\section{A. Hasman, J.L. Talmon}

Dept. of Medical Informatics, Maastricht University, Maastricht, The Netherlands

\title{
Research and Education
}

\section{Education and Research at the Department of Medical Informatics Maastricht}

\section{Introduction}

The department of Medical Informatics at Maastricht University was established in 1985 and serves the faculties of Medicine and of Health Sciences. We describe here the type of education that the department offers and discuss the research themes.

The department aims to contribute to the curriculum and conduct research in various areas of Medical Informatics such that future physicians will be better equipped in their decision-making process. With this broad mission in mind, the department contributes to a track devoted to the scientific education of students; research also focuses on electronic patient records and decision support.

\section{Education}

The educational system of the University of Maastricht is based on the concept of problem based learning (PBL) [1]. The aim of PBL is to overcome drawbacks in the traditional method of instruction, such as, the passive role of students during lectures, irrelevant subject matter in the curriculum, lack of integration between the disciplines, insufficient preparation of the students for continuing their postgraduate education and, of particular importance, the difficulties of graduates to apply in actual practice what they have learned.

The Maastricht system is characterized by self-study in which students organize their own learning process. Through consultation with lectures they determine priorities and choices in order to answer their own questions.

Students work in small tutorial groups (about 10 students), remaining together for one block period (4-6 weeks), meeting twice a week for 2 hours and with two days free for individual study. Group tasks provide the basis for the learning process, applying a sevenstep-approach to solve the tasks [1].

During each block, one theme related to subjects from different disciplines is discussed enabling students to experience the importance of interdisciplinary knowledge for the understanding of problems.

The department of Medical Informatics participates in a number of blocks in both faculties. In the Faculty of Medicine first year students are acquainted with the principles of searching the Web. Practicals deal with decision analysis, epidemiological concepts (e.g. prevalence, sensitivity, predictive value, and Bayes' rule), the application of protocols, etc.

In the Faculty of Health Sciences the department contributes to technology assessment, signal analysis, the electronic medical record, evaluation of clinical information systems, inno- vations of care, etc. [2,3]. Practicals deal with decision trees, Bayes' rule, simulation of bio-medical processes, simulation of workflow planning [4], etc. In addition, students gain skills in the use of Windows, Internet, word processing, databases and spreadsheets.

Students can opt to write a thesis on an information management subject, either by means of literature study but usually by conducting their own research for about six months.

The department also organizes several courses in health informatics [5]. Furthermore, department members were involved in defining(inter)national curricula in the Netherlands [6] and in Europe $[7,8]$. The department also contributes to the master courses in Medical Informatics given in Prague and Athens.

\section{Research}

In the Netherlands, academic research is largely concentrated in socalled research institutes. Individual departments can contribute to different research institutes. The research has to fit the goals of these institutes. Since we participate in different institutes our research was mainly focused on image analysis, decision support and the electronic patient record. These topics are described below. 
Table 1.

\begin{tabular}{|c|c|c|}
\hline 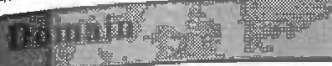 & 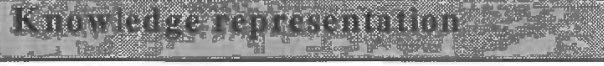 & 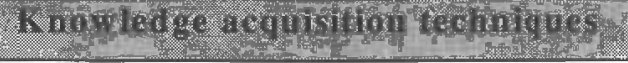 \\
\hline Vascular laboratory & Rules & Interviews/Delphi/induction \\
\hline Pathology & Word vectors & Techniques from information science \\
\hline$\overline{\text { Neurology }}$ & Rules & Interviews/Delphi \\
\hline Gastro-enterology & Rules/discriminant & Logistic regression/induction \\
\hline Family practice & Scoring system & Logistic regression \\
\hline Surgery & Rules & Simulation/induction \\
\hline General & Neural nets & Back-propagation \\
\hline Traumatology & Protocol & Interviews/protocol books \\
\hline
\end{tabular}

\section{Image analysis}

Several departments study cardiac mechanics in relation to ischemia. A prerequisite for such studies is accurate measurement of regional motion and deformation of the myocardium with sufficient resolution in space and time. Regional motion and deformation can be measured by following markers attached to the heart in a sequence of images. Several techniques allow to measure local deformation in closed-chest experiments. A method using radiopaque markers in combination with an X-ray stereocamera set-up was further developed. Radiopaque markers implanted in the myocardium serve as identifiable points to measure heart motion. With this technique accurate 3-D motion data of the heart with sufficient resolution in space and time in closed-chest dog experiments can be obtained, and the method can also detect deformation over more than one cardiac cycle, e.g., fordetecting gradual cardiac dilatation. or myocardial growth.

When a set of implanted radiopaque markers is observed in time by two cameras simultaneously in an X-ray stereo set-up, two sequences of video frames, each frame containing marker images, are obtained. After detection of the marker images a set of marker positions is available in each frame. From these data the marker trajectoriescan be reconstructed: the sequence of marker image positions in time.

When a large set of radiopaque markers is attached to the heart wall, their movements are not independent. Therefore, the movement of each marker can be represented as a weighted sum of common motion components with noise added to it. When the marker trajectories are represented by a matrix, a singular value decomposition (SVD) algorithm can be designed that removes noise while not detracting from the 'real' motion part. This results in more accurate position information.

The SVD model was also used to estimate missing data or to extrapolate a set of partly known marker tracks. It could be shown that by iteratively using the accurate extrapolations of partly known marker tracks which are obtained by the application of the SVD model, the markers can reliably and automatically be tracked despite complications caused by crossing tracks and missing marker image positions.

The results are described in several papers [9-11] and can be summarized as follows.

For a heart of diameter $70 \mathrm{~mm}$ with 20-100 markers attached to it, and using video image sequences of 50 frames (256 × 256 pixels, standard deviation marker position noise: $\mathbf{0 . 5}$ pixel) application of the described methods results in:

- a noise reduction of $50 \%-70 \%$, - correct automatic tracking of more than $90 \%$ of the markers, automatic identification of 20-70 stereo pairs of tracks (with the noise reduced to 0.25 pixel up to 100 stereo pairs of tracks can be correctly identified),

- a 3-D reconstruction error of 1-0.3 $\mathrm{mm}$.

The developed methods are generally applicable and have been successfully applied in several research areas:

- tracking distinct points (number: \pm 120 ) in tagging patterns when measuring deformation of the human heart by the use of MRI tagging techniques $[12,13]$,

- measuring 3-D deformation of hypertrophic dog hearts in long-term (several months) physiological experiments using 14 implanted radiopaque markers that are observed by X-ray [14],

- measuring 3-Ddeformation of skeletal muscle in physiological experiments using large amounts (70$150)$ of polystyrene optical markers $(0.5 \mathrm{~mm}$ diameter) attached to the outer surface of the muscle [15]

\section{Decision Support}

The decision-support research of the department focuses on four different topics:

- Interpretation of medical data

- Predictive models 
- Therapeutic advice

- Validation.

The investigations aim to assess the feasibility of several types of decisionsupport methods in a number of domains. Different knowledge acquisition techniques were also investigated.

We describe projects in each of these areas. Table 1 shows the domain in which the system was used, the knowledge representation and the knowledge acquisition techniques used.

\section{Interpretation of Medical Data}

This topic concerns the interpretation of digitally-stored medical data. Methods and systems are developed for this purpose.

\section{Vascular laboratory}

The first decision-support project was NIVTIS (Non-Invasive Vascular Test Interpretation System) a system for the interpretation of data collected in the vascular laboratory [16]. NIVTIS had to interpret both blood pressure (BP) data and the subjective interpretation of Doppler sounds recorded at the lower extremities. Also BP data recorded before and after a treadmill exercise had to be taken into account.

We interviewed technicians to gain knowledge on the interpretation of the data. The BP data recorded before and after exercise were a specific challenge as they are normally plotted on a graph and the resulting curve is described. To arrive at more objective criteria for interpretation two technicians graded 400 curves on a fivepoint scale, independently from each other. Cases on which they agreed were used to derive a classification tree from some parameters that characterized the curves. A non-parametric partitioning algorithm was used [17].

Cases that could not be correctly classified by the tree, as well as those on which the technicians disagreed, were resubmitted to the technicians for a second round of scoring. Feedback was given on the previous scorings. This procedure was repeated and after three rounds, the remaining discrepancies were discussed, resulting in 400 curves with an agreed upon grade. From this set a final classification tree was derived.

This project clearly demonstrated the value of Delphi-like techniques and the contribution of non-parametric data analysis to knowledge acquisition.

\section{Pathology}

In the Netherlands, pathology reports, coded in SNOMED, are centrally stored and managed. However, the codes are not always correct or complete and inter-individual differences between pathologists exist. To investigate this further we gave each of six groups of three pathologists a set of 40 pathology reports. Each report was accompanied by five different SNOMED code lines. The quality of each code line was rated by the three pathologists. Only in 54\% of the cases did all three experts give the same rating, indicating different opinions as to what constitutes correct and complete codes.

De Bruijn etal. compiled a database of coded pathology reports. Methods from information science were used to represent each report. All reports were transformed to a list of words. After all different words from all reports were determined, each report could be represented as a word vector. Each dimension of this vector indicates whether the word, that the dimension corresponds with, was indeed present in the report. The value can be either 1 (word present in the report) or 0 (word not present). The discriminative value of a word can be assessed by giving a word that appears in many other reports in the database a lower score. The similarity of two reports can be obtained by computing the in-product of the corresponding vectors. A newly entered report can then be compared to those already in the database. It was shown that in about $85 \%$ of the cases at least one report having a SNOMED code line appropriate for the new report, could be found among the five most similar reports $[18,19]$. When the code lines belonging to these five reports were presented to the pathologists the uniformity in coding increased by about $10 \%$.

\section{Surgery}

A non-parametric partitioning algorithm [17] was applied to determine the rules to be used in an intelligent alarm system. The goal of the study was to investigate whether the knowledge base of intelligent alarm systems can be obtained from mathematical models, as an alternative to elicit knowledge from human experts. As a medical problem domain, monitoring the mechanical ventilation of anesthetized patients was chosen, because several intelligent alarm systems exist in that area allowing to compare the performance of the new technique with those already developed. Twodifferent types of ventilator systems were modeled. Using the models, the signals usually measured at the ventilator could be obtained by simulation and the consequences of system faults (e.g. leaks and obstructions) could be studied. From the signals relevant parameters were derived, which were analyzed with the inductive machine learning program [17]. This resulted in a number of classification trees which were assessed using data obtained from 15 ventilated dogs and two goats. In the ventilators, leaks and obstructions were introduced. With the classification trees 93 to $99 \%$ of the mishaps were detected correctly with a minimal false positive error rate (mostly less than $0.2 \%$ ). It was concluded that rules determined from simulated signals are as good as the rules determined via animal experiments $[20,21]$. 


\section{Predictive Models}

Predictive models can be used to herease the efficiency of medical practice. By predicting the (normal) outcome of an endoscopic examination or a test on the basis of available patient data, the number of unnecessary examinations or tests may be reduced.

\section{Gastro-enterology}

The discriminative value of patient haracteristics and dyspeptic symptoms for upper gastrointestinal endoscopic diagnoses was studied to assess the possibility to predict the presence of clinically relevant endoscopic findings in patients referred for diagnostic upper gastrointestinal endoscopy. The possibility to distinguish patients with specific endoscopic diagnoses from those with a normal endoscopy was also investigated. Both logistic regression and a non-parametric partitioning algorithm [17] were applied to predict upper gastrointestinal endoscopic findings. It was concluded that it is possible to reduce the number of examinations by approximately $20 \%$ without missing any relevant endoscopic diagnoses based on the prediction of the endoscopic findings [2224].

\section{Family Practice}

Another study focused on the reduction of inadequate TSH test requests by GPs. The hypothesis was that patient characteristics would allow to predict whether the TSH test result would be positive or negative, thus avoiding TSH test requests that would produce negative results. A patient questionnaire containing 54 questions about signs and symptoms observed in thyroid disorders was filled in by 668 patients. Since the number of hypothyroid patients was too small, an instrument was developed that predicted the probability of a positive test result (for hyperthyroidism). Stepwise logistic regression was performed re- sulting in a regression coefficient for each relevant sign and symptom. On the basis of these coefficients a scoring system was developed. Only a limited number of signs and symptoms proved to be predictive for the test result.

The quality of the instrument was evaluated on the same population using a grouped Jackknife Method. With a sensitivity of $100 \%$ a test reduction of $24 \%$ was achieved [25].

\section{Therapy Advice}

\section{Traumatology}

Vissers et al. developed a passive automated protocol system ProtoVIEW for use in a traumatology department for treatment support. They investigated the effect of optional and mandatory protocol consultation on protocol adherence. It was shown that mandatory protocol consultation led to a $19 \%$ overall increase in protocol adherence. The diagnostic accuracy of both the intervention group (mandatory using ProtoVIEW) and the control group (optionally using a protocol book, containing the same information as ProtoVIEW) was equal (ProtoVIEW supported therapy and not diagnosis). Complete protocol adherence was in both groups higher for cases diagnosed correctly (59\% with computer support and $26 \%$ without) than for cases diagnosed incorrectly (35\% with and $24 \%$ without computer support). Complete protocol non-adherence was lower for cases diagnosed correctly ( $8 \%$ with and $7 \%$ without computer support) than for cases diagnosed incorrectly (35\% with and $48 \%$ without computer support). The residents involved in the study were also asked how certain they were about their diagnosis and treatment. Since residents were sure or very sure about $99 \%$ of their diagnoses and about $92 \%$ of their therapies it was concluded that a protocol system will not be consulted when the system is not integrated with an EPR system [26-28]. A direct coupling with an EPR was therefore needed for the ProtoVIEW system. Therefore, ProtoVIEW was upgraded making use of Web technology and is now a combination of an EPR and a protocol system [29].

\section{Neurology}

In another study an expert system was developed for prescribing antiepileptic drugs. To evaluate the prescriptions of the expert system a gold standard was needed. The prescriptions of five neurologists were used to determine a gold standard. It appeared that neurologists did not uniformly prescribe these drugs (kappa values < $0.4)$. The non-parametric partitioning algorithm was used to deduce the rationale of the neurologists when prescribing. The expert system was evaluated in several ways. It was concluded that the expert system was at least as good as each individual neurologist when the majority decision concerning the prescription was used as a gold standard [30].

\section{Validation}

A common issue in any research on ICT applications in medicine is their evaluation. Our department has been active in various EU-funded projects that developed and applied methodologies for evaluation of ICT, in particular of knowledge-based systems, in medicine [31-33].

In addition, we have co-responsibility for the ATIM and VATAM projects, also funded by the EU. The objective of the ATIM project was to make an inventory of existing methodologies, particularly in the domains of imaging workstations and knowledge-based systems. This has resulted in what is known as the "ATIM handbook" [34], which contains sections (written by various authors) dealing with method- 
ological issues and with assessment studies. Based on the results of ATIM, the VATAM project aimed to develop guidelines for assessment of ICT in health care. Eight projects from the Health Sector of the EU funded Telematics Application Programme of the $4^{\text {th }}$ framework program acted as users in the VATAM project. They defined their needs with respect to guidance in evaluation. It became clear that no single methodology can cover all aspects of applications of ICT in healthcare (the eight projects covered diverse issues such as EDI, middleware, tools for protocol based decision support, smart-card applications, clinical workstations).

The end result, which is also available on the VATAM webserver, is a checklist that will guide the user through a series of steps that address the issues that are relevant for setting up an assessment study [35].

In addition, the VATAM webserver contains a repository of information that is relevant for assessment studies [36]. This searchable repository contains definitions of terms related to assessment, description of assessment activities in projects, relevant literature, descriptions of tools for assessmentand descriptions of validated questionnaires that deal with e.g. the cognitive and ergonomic aspects of assessment. Visitors to this website can add information to this repository. The aim is to maintain and expand this website as an activity in IMIA's WG 15 on Technology Assessment

\section{The Electronic Patient Record}

The electronic patient record is an important research topic. Whereas other groups have focused on e.g. structured data entry, our studies initially focused on retrieval aspects. In particular, how searching information from medical narratives can be facili- tated by optimizing the structure of an EPR. An experimental EPR was developed allowing medical narratives to be viewed with different granularities. Three types of medical record structure were used in the study. A structure with coarse granularity which resembles the traditional medical record, presenting medical history, physical examination, and progress notes as undivided paragraphs. A structure with intermediate granularity, in which medical history and physical examination are divided according to organ-system and progress notes are partitioned into problem sections using subject headings. Finally a structure with fine granularity, in which medical history and physical examination are divided into single questions and examinations, and progress notes are, again, partitioned into problem sections.

Data of three patients were extracted from the paper record. Each patient was hospitalized for more than three weeks and at least 10 different active medical problems were recognized, of which at least three were manageable with only basic knowledge of internal medicine. In total 36 internists were asked to answer 19 questions about the three patients. The internists were given enough time to answer the questions. A cross-over design for three treatments was used to compare the effect of the three different granularities on the time needed to answer the questions. With the intermediate granularity the time needed for searching was reduced by $13 \%$ compared with the coarse granularity for medical history and physical examination. When comparing undivided progress notes with progress notes partitioned into problem sections a time gain of $22 \%$ was obtained. From the study it was concluded that context is very important when searching for information and that the use of headings corresponding to organ systems is more efficient than the use of headings that indicate each question or exami- nation result separately. When developing EPR systems, therefore, not only should the data entry forms be given appropriate attention but also the way in which the stored data are later presented to the user [37-39].

In this study the patient data were entered and presented in free text. When coupling decision-support systems with EPRs the use of a controlled vocabulary is indispensable. It is, however, still debated which data should be stored (should e.g. all basic data used by the physician to diagnose a patient be entered, or only the diagnosis and a few relevant data). Further research is needed to answer this question.

\section{Discussion}

We have explained the different approaches to education at our university and the role the Medical Informatics department plays in this educational environment. In addition, a number of our research projects have been presented. It should be noted that most of the studies were carried out in collaboration with a clinical counterpart.

As stated earlier, the research topics have to fit research areas defined by the research institutes. Because of this some other research topics are also pursued that differ in content from the above projects. One study, for example, dealt with decision support for planning clinical laboratory capacity [40]; in this case modeling and simulation is the main issue. Other topics include the development of a graphical browsing tool that supports information retrieval $[41,42]$, and the specification and assessment of methods supporting the development of neural networks in medicine [43-45]. We will not discuss these topics in detail but refer the reader to the literature for more information.

We decided to concentrate our future research mainly on the various 
spects of the EPR. Both the EPR isself and decision-support systems that an support physicians will be develped and their influence on the work of the physician will be studied.

From our research it can be conoluded that machine learning is a useful means to obtain rules that can be embedded in an expert system. Another issue is that physicians usually do not like pro-active systems, since these systems reduce the role of the physician to that of data entry. Decisionsupport systems should, therefore, be directly coupled to an EPR. Our current research deals with this topic.

\section{Acknowledgment}

The authors wish to thank all current and past members of the department for their contributions.

\section{References}

\section{Schmidt, H.G. Problem-based learning: ra-}

tionale and description. Education 1983;17:11-6.

2. Muijtjens AMM, Donkers HHLM, de Volder WCAM, et al A problem-oriented course in Medical Informatics. In: Van Bemmel JH, Zvárová J, eds. Knowledge, Information and Medical Education. Amsterdam: Elsevier Science Publishers, 1991:116-24.

3. Ambergen AW, Donker DK, Donkers HHLM, etal. Teaching medical informatics. In: Hansen R, Solheim BG, O'Moore RR, Roger FH, eds. Proceedings of Medical Informatics Europe '88. Heidelberg: Springer Verlag, 1998:311-5.

4. Groothuis $S$, van Merode GG. MEDMODEL in the health policy and management program at Maastricht University. Proc 1998 Western Multiconference 1998: 187-91.

5. Abraham I, Evers G, Hasman A, Tange $H$, Silkens R, Hein F. A summer institute on computer applications fornursing management: background, curriculum, and evaluation. J Cont Educ Nursing 1991;29:31-44.

6. Hasman A. Recommendations for medical informatics training in the Netherlands. Meth Inform Med 1994;33:308-11.

7. Hasman A. Education and training in health informatics: the IT-EDUCTRA project. Int J Med Inform 1998;50:179-86.

B. Hasman A. Education and health informatics. Int J Med Inform 1998;52:209-
16.

9. Muijtjens A, Roos JMA, Arts T, Hasman A, Reneman RS. Extrapolation of incomplete marker tracus by lower rank approximation. Int J Biomed Comput 1993;33:21939.

10. Muijtjens AMM, Roos JMA, Arts T, Hasman A, Reneman RS. Tracking markers with missing data by lower rank approximation. J Biomechanics 1997;30:95-8.

11. Muijtjens AMM, Roos JMA, Arts T, Hasman A. Maximum likelihood estimation in calibrating a stereo camera setup. Med Phys 1999;26:310-8.

12. Aelen FWL, Arts T, Sanders DGM, et al. Automated quantification of human left ventricular deformation withmagnetic resonance cardiac tagging. In: Murray A, Arzbaecher R, eds. Proc Int Conf Computers in Cardiology 1995. Piscataway, NJ: IEEE Inc, 1995:629-32.

13. Groot Koerkamp MAM, Snoep G, Muijtjens AMM, Kemerink GJ. Improving contrast and tracking of tags in cardiac magnetic resonance images. Magn Reson Med 1999;41:973-82.

14. Muijtjens AMM, Roos JMA, Van OMFM, et al. Measuring regional hypertrophy by radiopaque myocardial markers viewed in 3D with a single X-ray camera. In: Proc IEEE Conf Comput Cardiol 1997. The Institute of Electrical and Electronics Engineering, Inc., 1997:565-8.

15. Van DonkelaarCC, Willems PJB, Muijtjens AMM, Drost MR. Strain distribution at the surface of rat medial gastrocnemius muscle during contractions at different lengths. J Biomech 1999;32:755-62.

16. Schijven RAJ, Talmon JL, Penders R, Kitslaar PJEHM. Decision support system for the diagnosis and therapy planning in patients with peripheral vascular disease. In: Proceedings Computers in Cardiology 1987. The Institute of Electrical and Electronics Engineering, Inc., 1987:12932.

17. Talmon JL. A multiclass nonparametric partitioning algorithm. Pattern Recogn Letters 1986;4:31-8.

18. De Bruijn LM, Hasman A, Arends JW. Automatic SNOMED classification - a corpus-based method. Comput Meth Progr Bio 1997;54:115-22.

19. De Bruijn LM, Hasman A, Arends JW. Automatic coding of diagnostic reports. Meth Inform Med 1998;37:260-6.

20. Müller B, Hasman A, Blom JA. Building intelligent alarm systems by combining mathematical models and inductive machine learning techniques. Int J Biomed Comput 1997;41:107-24.

21. Müiller B, Hasman A, Blom JA. Building intelligent alarm systems by combining mathematical models and inductive ma- chine learning techniques: Part2-Sensitivity analysis. Int $\mathrm{J}$ Biomed Comput 1997:42:165-79.

22. Adang RP, Vismans FJE, Talmon J, Hasman A, Ambergen AW, Stockbrugger $R W$. The diagnostic outcome of upper gastrointestinal endoscopy: are referral source and patient age determining factors? Eur J Gastroenterol Hepat 1994;6:329-35.

23. Adang RP, Vismans FJE, Talmon JL, Hasman A, Ambergen AW, Stockbrugger RW. Appropriateness of indications for diagnostic upper gastrointestinal endoscopy: association with relevant endoscopic disease. Gastrointest Endoscopy 1995;42:390-97.

24. Adang RP, Ambergen AW, Talmon JL, Hasman A, Vismans JFE, Stockbrugger $\mathrm{RW}$. The discriminative value of patient characteristics and dyspeptic symptoms for upper gastrointestinal endoscopic diagnoses: A study on the clinical presentation of 1,147 patients. Digestion 1996;57:11834.

25. Dupuits FMHM, Pop P, Hasman A, Schouten HJA. A rational request behavior: The development of prediction instruments regarding thyroid function tests in primary care. Meth Inform Med 1999;38:31-6.

26. Vissers M, Hasman A, Van der Linden CJ. Consultation behaviour of residents supported with a protocol processing system (ProtoVIEW) at the emergency ward. Int J Biomed Comput 1995;38:181-7.

27. Vissers M, Biert J, van der Linden CJ, Hasman A. Effects of a supportive proto col processing system (ProtoVIEW) on clinical behaviour of residents in the Accident and Emergency department. Comp Meth Prog Bio 1996;49:177-84.

28. Vissers $M$, Hasman A, van der Linden CJ. Impact of a protocol processing system (ProtoVIEW) on clinical behaviour of residents and treatment. Int J Biomed Comput 1996;42:143-50.

29. Vissers MC, Hasman A. Building a flexible protocol information system with 'ready for use' web-technology. Int I Med Inform 1999;53:163-74.

30. Smeets RPAM, Meinardi H, Talmon JL, Hasman A. Agreement and correctness in adjusting antiepileptic drug treatment: a need for rationaldrug treatment? Epilepsia 1999;40: 763-8.

31. Clarke K, O'Moore R, Smeets R, Talmon $\mathrm{JL}$, Brender, McNair P, Nykänen $\mathrm{P}$, Grimson J, Barber B. A methodology for evaluation of knowledge-based systems in medicine. Artif Intell Med 1994;6:107-21.

32. Brender ., Talmon JL, Nykänen ., McNair P, Demeester M, Beuscart R. On the evaluation of systemintegration. In: Van Gennip EMSJ, Talmon JL, eds. Assessment and 
Evaluation of Information Technologies. Amsterdam: IOS Press, 1995:189-208.

33. Vissers MC, Talmon JL. Evaluating the MACRO solution. In: Pappas C, Maglaveras N, Scherrer J-R, eds. Proceedings of Medical Informatics Europe '97. Amsterdam: IOS Press, 1997:741-5.

34. Gennip EMSJ van, Talmon JL, eds. Assessment and Evaluation of Information Technologies. Amsterdam: IOS Press, 1995.

35. TalmonЛ, Enning J, Castãneda G, Eurlings F, Hoyer D, Nykänen P, Sanz F, Thayer C, Vissers M: The VATAM guidelines. Int J Med Inform 1999, in press.

36. http://www-vatam.unimaas.nl

37. Tange HJ. How to approach the structering of the medical record?: Towards amodel for flexible access to free text medical data. Int J Biomed Comput 1996;42:27-34.

38. Tange HJ, Dreessen VAB, Hasman A, Donkers HHLM. An experimental electronic medical-record system with multiple views on medical narratives. Comput Meth Progr Bio 1997;54:157-72.

39. Tange HJ, Schouten HC, Kester A, Hasman
A. The granularity of medical narratives and its effect on the speed and completeness of information retrieval. J Am Med Inform Assoc 1998;5:571-82.

40. Merode GG van, Hasman A, Derks J, Schoenmaker B, Goldschmidt HMJ. Advanced management facilities for clinical laboratories. Comput Meth Progr Bio 1996;50:195-205.

41. Wiesman F, Hasman A. Graphical information retrieval by browsing meta-information. Comput Meth Progr Bio 1997;53:13552.

42. Dietz JLG, van der Pol $R$, Wiesman F. The ARCHIMEDES network system: a system for searching and accessing information in multiple multimedia sources. J Intel Inform Syst 1997;8:77-101.

43. Egmont-Petersen M, Talmon JL, Brender J, McNair P. On the quality of neural net classifiers. Artif Intell Med 1994;6:359 81.

44. Egmont-Petersen M, Talmon JL, Hasman A. Robustness metrics for measuring the influence of additives on the performance of statistical classifiers. Int J Med Inform
1997;46:103-12.

45. Egmont-Petersen M, Talmon JL, Hasman A, Ambergen AW. Assessing the importance of features formulti-layerperceptrons. Neural Networks 1998; 11: 623-35.

Address of the authors:
A. Hasman, J.L. Talmon,
Department of Medical Informatics, Maastricht University,
PO Box 616,
6200 MD Maastricht, The Netherlands
e-mail: hasman@mi.unimaas.nl 